\title{
Identification and functional analysis of the cyclopropane fatty acid synthase of Brucella abortus
}

Correspondence

Maite Iriarte

miriart@unav.es

Received 28 October 2011

Revised 23 December 2011

Accepted 17 January 2012

\author{
Leyre Palacios-Chaves, ${ }^{1}$ Amaia Zúñiga-Ripa, ${ }^{1}$ Ana Gutiérrez, ${ }^{2}$ \\ Yolanda Gil-Ramírez, ${ }^{1}$ Raquel Conde-Álvarez, ${ }^{1}$ Ignacio Moriyón ${ }^{1} \dagger$ \\ and Maite Iriarte ${ }^{1} \dagger$ \\ ${ }^{1}$ Instituto de Salud Tropical y Departamento de Microbiología y Parasitología, \\ Universidad de Navarra, Pamplona, Spain \\ ${ }^{2}$ Instituto de Recursos Naturales y Agrobiología de Sevilla, Consejo Superior de Investigaciones \\ Científicas, Seville, Spain
}

The brucellae are facultative intracellular pathogens of mammals that are transmitted by contact with infected animals or contaminated materials. Several major lipidic components of the brucella cell envelope are imperfectly recognized by innate immunity, thus contributing to virulence. These components carry large proportions of acyl chains of lactobacillic acid, a long chain cyclopropane fatty acid (CFA). CFAs result from addition of a methylene group to unsaturated acyl chains and contribute to resistance to acidity, dryness and high osmolarity in many bacteria and to virulence in mycobacteria. We examined the role of lactobacillic acid in Brucella abortus virulence by creating a mutant in ORF BAB1_0476, the putative CFA synthase gene. The mutant did not incorporate $\left[{ }^{14} \mathrm{C}\right]$ methyl groups into lipids, lacked CFAs and synthesized the unsaturated precursors, proving that BAB1_0476 actually encodes a CFA synthase. BAB1_0476 promoter-luxAB fusion studies showed that CFA synthase expression was promoted by acid $\mathrm{pH}$ and high osmolarity. The mutant was not attenuated in macrophages or mice, strongly suggesting that CFAs are not essential for $B$. abortus intracellular life. However, when the mutant was tested under high osmolarity on agar and acid $\mathrm{pH}$, two conditions likely to occur on contaminated materials and fomites, they showed reduced ability to grow or survive. Since CFA synthesis entails high ATP expenses and brucellae produce large proportions of lactobacillic acyl chains, we speculate that the CFA synthase has been conserved because it is useful for survival extracellularly, thus facilitating persistence in contaminated materials and transmission to new hosts.

\section{INTRODUCTION}

Brucellosis is a zoonosis of great importance that has a heavy impact in developing countries. The causative agents belong to the genus Brucella, a group of $\alpha-2$ Proteobacteria characterized by their ability to multiply in macrophages, dendritic cells and other cells of a variety of mammals. Although the brucellae do not multiply outside their hosts under natural conditions, they can grow in laboratory axenic cultures and persist in the soil and contaminated materials for weeks or even months depending upon the conditions (Huddleson, 1943; Corbel, 2006). The cell envelope plays an essential role in brucella virulence. This structure displays a

Abbreviations: C17cyc, cis-9,10-methylene hexadecanoic acid; C19cyc, cis-11,12-methylene-octadecanoic; CFA, cyclopropane fatty acid; PAMP, pathogen-associated molecular pattern; SAM, S-adenosyl-Lmethionine.

†These authors contributed equally to this work. number of features that hinder recognition by innate immunity, including high stability when confronted with bactericidal peptides and poorly marked pathogen-associated molecular patterns (PAMPs) in phospholipids, ornithine lipids, lipopolysaccharides and lipoproteins (Barquero-Calvo et al., 2007; Freer et al., 1996; Martínez de Tejada et al., 1995; Martínez de Tejada \& Moriyón, 1993; Moriyón \& Berman, 1982; Palacios-Chaves et al., 2011). Interestingly, all these lipidic components carry large proportions of lactobacillic acyl chains (Gómez-Miguel \& Moriyón, 1986; Thiele \& Schwinn, 1973; Velasco et al., 2000). Lactobacillic acid [cis11,12-methylene-octadecanoic (C19cyc)] is a long chain cyclopropane fatty acid (CFA) and, although CFAs are produced by other bacteria under unfavourable conditions, the most common ones [cis-9,10-methylene hexadecanoic acid (C17cyc) and other C17 CFAs] have shorter acyl chains.

CFAs result from the modification of pre-existing unsaturated acyl chains by addition of a methylene group to the 
carbon-carbon double bonds (Wang \& Cronan, 1994). This reaction is carried out by soluble CFA synthases that use $S$-adenosyl-L-methionine (SAM) as the methylene donor. CFA synthesis typically starts at the onset of the stationary phase of growth and, consistent with this, it has been proposed that CFAs contribute to adaptation of the envelope to unfavourable conditions, including low oxygen tension, nutrient limitation, hydrogen peroxide, acid $\mathrm{pH}$, desiccation and high ionic strength and/or osmolarity. Moreover, several pathogenic Mycobacterium species carry CFA synthases and methyltransferases that alter the PAMP of mycolic acids and are thus important in hampering innate immunity recognition (Barkan et al., 2010; Dao et al., 2008; Glickman et al., 2000; Rao et al., 2005, 2006). It is, therefore, conceivable that lactobacillic acid could play a role in brucella virulence by contributing to adapting the envelope to intracellular environments, as proposed by Roop et al. (2003) or to reduce the PAMP of envelope molecules. In this work, we report on the identification of the gene coding for the B. abortus CFA synthase and on the effects of its dysfunction.

\section{METHODS}

Bacterial strains and growth conditions. Brucella abortus 2308 $\mathrm{Nal}^{\mathrm{R}}$ (BAB-parental) is a smooth virulent strain used in previous studies (Conde-Álvarez et al., 2006; Palacios-Chaves et al., 2011). This strain and the mutant, revertant and complemented strains resulting from the genetic manipulations described below were characterized following standard Brucella typing procedures (colony morphology, crystal violet exclusion, catalase, oxidase, urease and acriflavine agglutination tests, sensitivity to $\mathrm{Tb}, \mathrm{Wb}, \mathrm{Iz}$ and $\mathrm{R} / \mathrm{C}$ phages, agglutination with anti-A and anti-M monospecific sera, $\mathrm{CO}_{2}$ and serum dependence, and susceptibility to thionine blue, fuchsine and safranin) (Alton et al., 1988). Bacteria were routinely grown in tryptic soy broth (TSB) (bioMérieux) or on tryptic soy agar (TSA) either plain or supplemented with $25 \mu \mathrm{g}$ nalidixic acid (Nal) $\mathrm{ml}^{-1}$ and/or $50 \mu \mathrm{g}$ kanamycin $(\mathrm{Km}) \mathrm{ml}^{-1}$ and/or $20 \mu \mathrm{g}$ chloramphenicol $(\mathrm{Cm})$ $\mathrm{ml}^{-1}$ (Sigma Aldrich) and/or $5 \%(\mathrm{w} / \mathrm{v})$ sucrose.

DNA manipulation, construction of mutants and complementation. Plasmid and chromosomal DNA were extracted with QIAprep spin miniprep (Qiagen) and ultraclean microbial DNA isolation kit (Mo Bio Laboratories), respectively. When necessary, DNA was purified from agarose gels using a QIAquick gel extraction kit (Qiagen). DNA sequencing was performed at the Servicio de Secuenciación de DNA of the Center for Applied Medical Research (University of Navarra, Pamplona, Spain). Primers were synthesized by Sigma Aldrich. DNA and protein homology searches were carried out using the NCBI (http://www.ncbi.nlm.nih.gov) and the EMBLEuropean Bioinformatics Institute (http://www.ebi.ac.UK/ebi_home. html) servers. Sequences were obtained from the KEGG (http://www. genome.jp/kegg/) and NCBI (http://www.ncbi.nlm.nih.gov/genomes/ lproks.cgi?view $=1$ ) databases.

To construct an in-frame mutant in ORF BAB1_0476 [putative $B$. abortus cfa (cyclopropane fatty acid synthase) gene], oligonucleotides BAB1_0476-F1 (5'-CGGTTGATTGACGGAAAACT-3') and BAB1_ 0476-R2 (5'-GTTAAACGCAACGGCTCTTT-3') were used to amplify a 286 bp fragment including codons $1-54$ plus 124 nt upstream of the start codon, and oligonucleotides BAB1_0476-F3 (5' -AAAGAGCCGTTGCGTTTAACTATATCGAGGCGGAGGAAAA-3') and BAB1_0476R4 (5'-TCCGCCATGATTCTCTTTTC- $\left.3^{\prime}\right)$ were used to amplify a
$305 \mathrm{bp}$ fragment including codons 395-426 of the ORF and $206 \mathrm{bp}$ downstream of the stop codon. These fragments were ligated by overlapping PCR using oligonucleotides BAB1_0476-F1 and BAB1_0476-R4 for amplification, and the complementary regions of BAB1_0476-R2 and BAB1_0476-F3 for overlapping. The resulting fragment, containing the BAB1_0476 deletion allele, was cloned into pCR2.1 (Invitrogen) to generate plasmid pLPI-3, sequenced to ensure maintenance of the reading frame, and subcloned into the BamHI and XbaI sites of the suicide plasmid pJQK (Scupham \& Triplett, 1997). The resulting suicide mutator plasmid (pLPI-4) was introduced into BABparental by conjugation (Conde-Álvarez et al., 2006; Palacios-Chaves et al., 2011); integration of this suicide vector was selected by $\mathrm{Nal}$ and $\mathrm{Km}$ resistance and the excisions [generating both $c f a$ mutant $(\mathrm{BAB} \Delta c f a)$ and revertant (BAB-revertant) strains recovering the intact gene] were selected by $\mathrm{Nal}$ and sucrose resistance and $\mathrm{Km}$ sensitivity. The resulting colonies were screened by PCR with primers BAB1_0476-F1 and BAB1_0476-R4 which amplify fragments of 591 or 1611 bp from the deletion mutants and the revertant strains, respectively. The mutation resulted in the loss of both the SAM-methyltransferase consensus motif (VLE/DXGXGXG) and the amino acids equivalent to those responsible for the enzymic activity of the Escherichia coli CFA synthase (C139, E239, H266, I268 and Y317) (Courtois et al., 2004; Ingrosso et al., 1989).

For complementation, a plasmid carrying ORF BAB1_0476 was constructed using the Gateway cloning Technology (Invitrogen). Since the sequences of BAB1_0476 and of its Brucella melitensis homologue are $99 \%$ identical, the clone carrying BMEI_1484 was taken from the B. melitensis ORFeome (Dricot et al., 2004) and subcloned into plasmid pRH001 (Hallez et al., 2007) to produce plasmid pLPI-15. This plasmid was introduced into the BAB $\Delta c f a$ mutant by mating with E. coli S17 $\lambda$ pir and the conjugants (BAB $\Delta c f a$ pLPI-15) were selected by plating onto TSA-Nal-Cm plates.

Gas chromatography-MS analysis. For fatty acid analysis, dry bacteria $(150 \mathrm{mg})$ were treated with $15 \% \mathrm{NaOH}(3.75 \mathrm{ml})$ in $50 \%(\mathrm{v} /$ v) methanol for $30 \mathrm{~min}$ at $100{ }^{\circ} \mathrm{C}$. The saponified material was cooled and acidified with $\mathrm{HCl}$, extracted three times with hexane-methyl tertbutyl ether $(1: 1)$, and the pooled extracts were evaporated to dryness and dissolved in chloroform. The analyses were performed with a Varian 3800 chromatograph coupled to an ion-trap detector (Varian 4000) using a fused-silica DB-5HT capillary column $(12 \mathrm{~m} \times 0.25 \mathrm{~mm}$ internal diameter, $0.1 \mu \mathrm{m}$ film thickness) from J\&W Scientific. The oven was heated from 50 to $90{ }^{\circ} \mathrm{C}$ at $30{ }^{\circ} \mathrm{C} \mathrm{min}{ }^{-1}$, and from 90 to $250{ }^{\circ} \mathrm{C}$ at $8{ }^{\circ} \mathrm{C} \mathrm{min}^{-1}$. The transfer line was kept at $300{ }^{\circ} \mathrm{C}$ and the injector temperature was set at $250^{\circ} \mathrm{C}$. Helium was used as carrier gas at a rate of $2 \mathrm{ml} \mathrm{min}^{-1}$ and the injection was performed in the splitless mode. Trimethylsilyl-diazomethane methylation and bis (trimethylsilyl)trifluoroacetamide silylation in the presence of pyridine were used to produce the appropriate derivatives. Compounds were identified by mass fragmentography and by comparing their mass spectra with those of the Wiley and NIST libraries.

$\left[{ }^{14} \mathrm{C}\right]$ SAM labelling of free lipids. Saline-washed stationary phase bacteria grown in TSB were adjusted to $\mathrm{OD}_{600} 1.0$, and $1 \mathrm{ml}$ of this suspension was inoculated into $10 \mathrm{ml}$ minimal Gerhardt's medium (Gerhardt, 1958). Immediately, $1 \mathrm{ml}$ of the culture was supplemented with $0.5 \mu \mathrm{Ci}\left(1.85 \times 10^{4} \mathrm{~Bq}\right)\left[{ }^{14} \mathrm{C}\right] \mathrm{SAM}\left(56 \mathrm{mCi} \mathrm{mmol}^{-1}\right)$ (Hacker et al., 2008). After incubation for $24 \mathrm{~h}$, bacteria were inactivated with phenol $(0.5 \%, \mathrm{v} / \mathrm{v})$, harvested by centrifugation, washed with $500 \mu \mathrm{l}$ water and resuspended in $100 \mu \mathrm{l}$ water. Lipids were then extracted with chloroform: methanol (Bligh \& Dyer, 1959), resolved on silica gel 60 high-performance TLC plates (Merck Chemicals) using n-propanol/ propionic acid/chloroform/water $(3: 2: 2: 1)$ and developed by autoradiography and charring with $15 \%(\mathrm{v} / \mathrm{v})$ sulfuric acid in ethanol.

CFA synthase gene expression studies. To construct a BAB1_0476 promoter-luxAB transcriptional fusion, a fragment 
containing the ATG of BAB1_0476 plus 200 bp upstream of this start codon was amplified using primers BAB1_0476-F11 (5' -GGGATCCAAATTTCGGGCGGTCGCTATTGGA-3'; BamHI site underlined) and BAB1_0476-R12 (5'-TTCTAGACATCGGCCCCATCTCCTCTTTGGGAA-3'; Xbal site underlined), cloned into pGEM-T Easy (Promega) and subcloned (using the BamHI and XbaI sites) into pSKoriTKmLuxAB. The resulting plasmid was then transferred to B. abortus (Conde-Álvarez et al., 2006; Palacios-Chaves et al., 2011) and the clones carrying the transcriptional fusion [integrated into chromosome I ( $B$. abortus pBAB1_0476-luxAB)] selected by $\mathrm{Nal}$ and $\mathrm{Km}$ resistance and by PCR using oligonucleotides BAB1_0476-F11 and $l u x A B-R v\left(5^{\prime}\right.$ AGCAACCAAACGGTGTCGAA-3'). To measure luciferase activity, fresh $B$. abortus pBAB1_0476-luxAB cells were adjusted to $\mathrm{OD}_{600} 0.4$ in saline and $50 \mu \mathrm{l}$ was inoculated into $10 \mathrm{ml}$ TSB, TSB adjusted to $\mathrm{pH} 6$ with Mcllvaine's buffer $\left(0.2 \mathrm{M} \mathrm{Na}_{2} \mathrm{HPO}_{4} ; 0.1 \mathrm{M}\right.$ citric acid $)$ or TSB$340 \mathrm{mM}$ sucrose (in this case, a $200 \mu \mathrm{l}$ inoculum was used to correct for the longer lag phase). Growth was followed by measuring $\mathrm{OD}_{600}$ and $1 \mathrm{ml}$ aliquots were taken periodically to measure the luminescence in relative units of luminescence (RLU) after addition of $100 \mu \mathrm{l}$ ethanol/decanal $(1: 1)$.

Effect of acid pH on bacterial survival. Bacteria were grown in TSB up to the stationary phase, harvested by centrifugation, washed twice and adjusted to $\mathrm{OD}_{600} 0.9$ with saline. Then, $500 \mu \mathrm{l}$ was suspended in $10 \mathrm{ml}$ McIlvaine's buffer adjusted to the appropriate $\mathrm{pH}$ and incubated at $37{ }^{\circ} \mathrm{C}$ with shaking (130 r.p.m.). After 48, 72, 96 and $120 \mathrm{~h}, 100 \mu \mathrm{l}$ aliquots were serially diluted and plated onto TSB agar plates that were incubated at $37^{\circ} \mathrm{C}$ for up to 6 days.

Effect of osmolarity on bacterial growth. Luria-Bertani broth (LB) $[1 \%(\mathrm{w} / \mathrm{v})$ tryptic casein $-0.5 \%(\mathrm{w} / \mathrm{v})$ yeast extract; Becton Dickinson) was used, either plain or supplemented with $340 \mathrm{mM}$ sucrose (LB-340 mM sucrose). Bacteria were grown in LB up to stationary phase, harvested by centrifugation, washed twice and adjusted to $\mathrm{OD}_{600} 0.17$ with saline. An aliquot $(10 \mu \mathrm{l})$ of this suspension was inoculated into $190 \mu \mathrm{LB}$ or LB-340 mM sucrose and incubated in a Bioscreen C (Bioscreen Instruments) at $37{ }^{\circ} \mathrm{C}$ with continuous stirring. For studies on solid media, bacteria were suspended to $\mathrm{OD}_{600} 0.9$, serially diluted, and $10 \mu \mathrm{l}$ was spotted on LB agar or LB-agar- $340 \mathrm{mM}$ sucrose; the plates were incubated at $37^{\circ} \mathrm{C}$ for 6 days.

Multiplication in murine macrophages. Murine RAW 264.7 macrophages (ATCC TIB-71) were cultured in Dulbecco's modified Eagle's medium [DMEM, (Gibco; Invitrogen)] supplemented with $10 \%(\mathrm{v} / \mathrm{v})$ fetal bovine serum, $1 \%(\mathrm{v} / \mathrm{v})$ non-essential amino acids $100 \times$ (Gibco; Invitrogen) and $1 \%$ (v/v) L-glutamine $200 \mathrm{mM}$ (Sigma Aldrich) under a $5 \% \mathrm{CO}_{2}$ atmosphere. Infections were performed using an m.o.i. of 50:1 by centrifuging bacteria onto macrophages (400 $\mathrm{g}$ for $10 \mathrm{~min}$ at $4{ }^{\circ} \mathrm{C}$ ), followed by incubation at $37^{\circ} \mathrm{C}$ for $30 \mathrm{~min}$ under $5 \% \mathrm{CO}_{2}$. To remove extracellular bacteria, macrophages were extensively washed with DMEM and incubated in medium with $100 \mu \mathrm{g}$ gentamicin $\mathrm{ml}^{-1}$ for $60 \mathrm{~min}$. Thereafter, the antibiotic concentration was decreased to $25 \mu \mathrm{g} \mathrm{ml}^{-1}$. After 2, 24 and $48 \mathrm{~h}$, infected macrophages were washed three times with $100 \mathrm{mM}$ PBS ( $\mathrm{pH} 7.2$ ), lysed with $0.1 \%(\mathrm{v} / \mathrm{v})$ Triton X-100 in PBS and serial dilutions plated onto TSA to enumerate the c.f.u. The attenuated $B$. abortus virB mutant was used as a control (Sieira et al., 2000).

Virulence in mice. Groups of 7 -week-old female $B A L B / c$ mice (Charles River) were inoculated intraperitoneally with $1.87 \times 10^{4}$ c.f.u. $\mathrm{BAB} \Delta c f a$ per mouse or $6.83 \times 10^{4}$ c.f.u. BAB-parental per mouse in $0.1 \mathrm{ml}$ PBS. Spleen weights (mean \pm SD, g per spleen) and c.f.u. (mean \pm sD, $\log _{10}$ c.f.u. per spleen) were determined in five mice 2 and 8 weeks after inoculation and the identity of the bacterial isolates was confirmed by PCR. The experimental procedures (i.e. preparation and administration of inocula, assessment of the inoculating doses and determination of the number of c.f.u. per spleen) have been described previously (Grilló et al., 2006). Statistical comparisons between means were performed by ANOVA and Fisher's protected least significant differences tests (Grilló et al., 2006). Animal handling and related procedures were in accordance with the current European legislation (directive 86/609/EEC) and approved by the Animal Welfare Committee of the institution (protocol no. R102/2007).

\section{RESULTS}

\section{ORF BAB1_0476 encodes a CFA synthase}

The genome of B. abortus 2308 (http://www.ncbi.nlm.nih. gov/genomes/lproks.cgi) contains an ORF (BAB1_0476) annotated as encoding a methyltransferase or a CFA synthase. Since some bacteria have more than one of these enzymes, we searched for orthologues of the three CFA synthases annotated in Mesorhizobium loti (Kaneko et al., 2000) and of the $c f a-1$ and $c f a-2$ CFA synthase genes described in Sinorhizobium meliloti (Saborido Basconcillo et al., 2009) as well as for homologues of the methyltransferases that modify Mycobacterium tuberculosis mycolic acids (Dao et al., 2008; Rao et al., 2005, 2006). Although we identified several methyltransferase homologues in B. abortus, only ORF BAB1_0476 had a significant homology with CFA synthases. Moreover, the predicted protein conserves both the SAMbinding consensus motif (VLE/DXGXGXG) and the amino acids equivalent to the C139, E239, H266, I268 and Y317 residues that are essential for the activity of the E. coli phospholipid CFA synthase (Courtois et al., 2004; Ingrosso et al., 1989). ORF BAB1_0476 has identical orthologues in the reference strains of all $B$. abortus and Brucella suis biovars as well as in vaccine B. abortus S19, B. ovis and Brucella ceti. A deletion removing an amino acid outside the predicted functional sections exists in the reference strains of all $B$. melitensis biovars, vaccine $B$. melitensis Rev 1 and $B$. neotomae. Also, there is a 2 aa deletion in Brucella pinnipedialis (http://www.broadinstitute.org/annotation/ genome/brucella_group/).

To analyse the activity of the protein encoded by BAB1_0476, we constructed a B. abortus $2308 \mathrm{Nal}^{\mathrm{R}}$ (BABparental) BAB1_0476-deleted mutant by making an in-frame deletion encompassing the SAM-binding consensus motif and the C139, E239, H266, I268 and Y317 equivalents. The mutant was identical in colony morphology and growth, as well as in all standard tests used to type smooth brucellae, to the parental strain (Alton et al., 1988). Then, we analysed the fatty acids of stationary phase cells of BAB-parental, BAB $\Delta c f a$ and BAB $\Delta c f a-p L P I-15$ (BAB $\Delta c f a$ complemented strain). As can be seen in Fig. 1, the major fatty acid in BAB-parental was lactobacillic acid (C19cyc). Palmitic (C16:0), cis-9,10methylene hexadecanoic $(\mathrm{C} 17 \mathrm{cyc})$, stearic $(\mathrm{C} 18: 0)$, vaccenic [C18:1(n-7), the C19cyc precursor] acid and trace amounts of palmitoleic [C16:1(n-7), the C17cyc precursor] acid were also present. In contrast, mutant BAB $\Delta c f a$ lacked CFAs and showed increased amounts of cyclopropane acid precursors, vaccenic in particular (Fig. 1). Complementation restored 

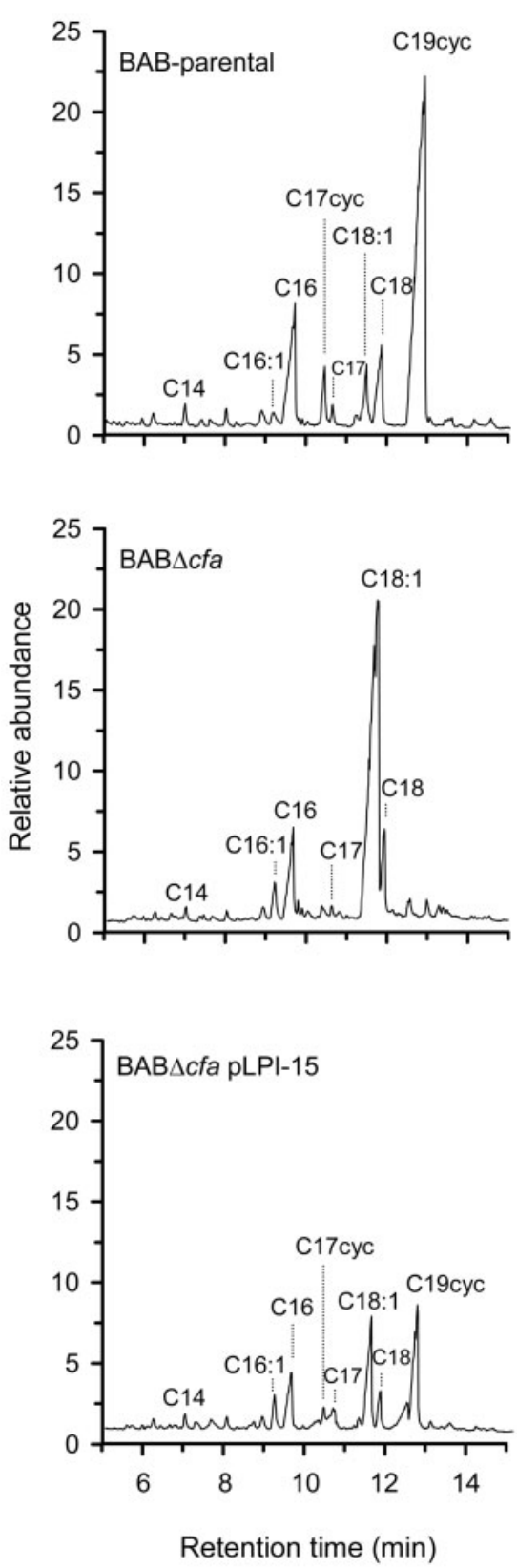

Fig. 1. Gas chromatography-MS analysis of fatty acids (as methyl esters) from $B A B$-parental, $B A B \Delta c f a$ mutant and $B A B \Delta c f a$ mutant carrying plasmid pLPI-15 encoding the complete ORF BAB1_0476.

the ability to generate CFAs, although not to the level of the parental strain (Fig. 1).

To extend these results, we tested the ability of BAB-parental and $\mathrm{BAB} \Delta c f a$ to incorporate methylene groups into lipids using SAM as the donor. To this end, we used a minimal medium supplemented with $\left[{ }^{14} \mathrm{C}\right] \mathrm{SAM}$ and extracted the free lipids of stationary phase bacteria. TLC followed by autoradiography showed that whereas the ornithine lipids

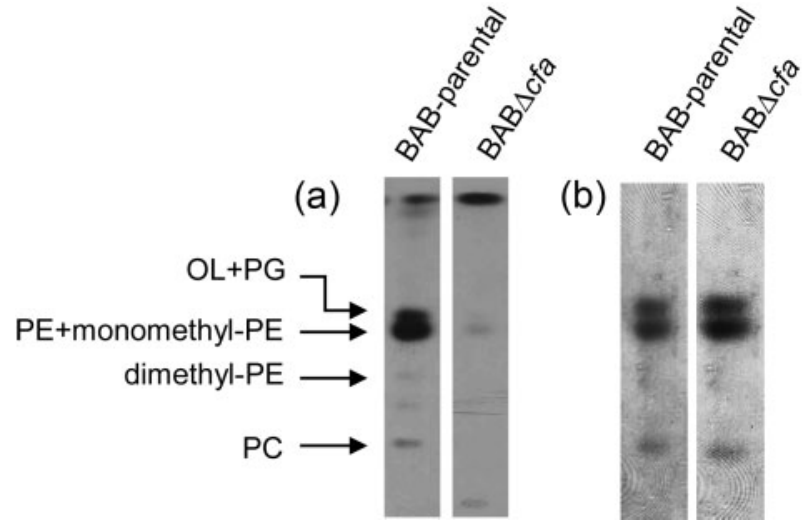

Fig. 2. B. abortus ORF BAB1_0476 encodes a protein with methyltransferase activity. TLC analysis of the free lipids of BABparental and BAB $\Delta$ cfa grown in Gerhardt's minimal medium supplemented with $\left[{ }^{14} \mathrm{C}\right] \mathrm{SAM}$. (a) Autoradiography and (b) charring. OL, Ornithine lipids; PG, phosphatidylglycerol; PE, phosphatidylethanolamine; PC, phosphatidylcholine.

and major phospholipids of BAB-parental were labelled, those of BAB $\Delta c f a$ were not (Fig. 2). Altogether, the results of the fatty acid analysis and $\left[{ }^{14} \mathrm{C}\right] \mathrm{SAM}$ experiments clearly indicate that BAB1_0476 encodes a CFA synthase and that no other ORF is involved in CFA synthesis under these growth conditions. Accordingly, the name $c f a$ was used for BAB1_0476.

\section{Expression of $B$. abortus cfa occurs during the exponential phase of growth and increases in acid or hyperosmotic media but it is not involved in virulence}

Since brucella temporarily passes through an acidic intracellular vesicle before reaching the intracellular replicative niche, it has been proposed that CFAs play a role in resistance to this unfavourable milieu (Roop et al., 2003). Similarly, the periplasmic cyclic glucans that act as a brucella virulence factor are osmoregulated (Arellano-Reynoso et al., 2005; Iñón de Iannino et al., 2000; Roset et al., 2006), suggesting osmotic changes during intracellular life. Thus, we studied the influence of $\mathrm{pH}$ and osmotic tension on $B$. abortus $c f a$ expression in vitro using a $c f a$ (BAB1_0476) promoter-luxAB transcriptional fusion. Fig. 3 shows that there was transient $c f a$ promoter activity in TSB at $\mathrm{pH} 7$ and a threefold increase in promoter activity at $\mathrm{pH} 6$ or in $340 \mathrm{mM}$ sucrose during the exponential growth phase. Then, we tested the virulence of $\mathrm{BAB} \Delta c f a$ in macrophages and mice. Fig. 4(a) shows that, in contrast with the decreased viability of the virB mutant (Sieira et al., 2000) used as a reference of attenuation, BAB-parental and $B A B \Delta c f a$ multiplied similarly in murine RAW 264.7 macrophages. Likewise, we did not find differences in multiplication and persistence in the spleens (Fig. 4b) or in spleen weights (not shown) of intraperitoneally inoculated BALB/c mice, 


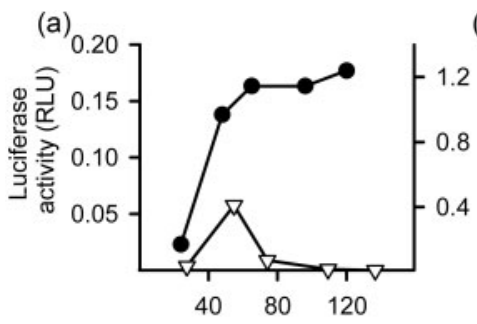

(b)
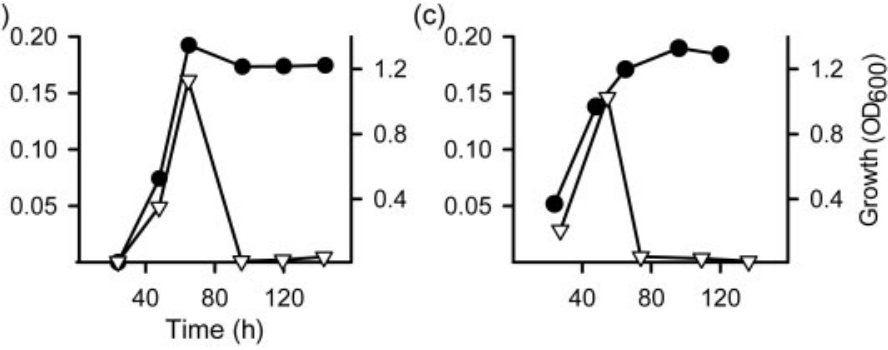

Fig. 3. Activity of the $B$. abortus cfa promoter during growth under standard (a), acidic (b) and hyperosmotic (c) conditions. The results show the luciferase activity $[\nabla$; in relative luminiscence units (RLU)] of a $B$. abortus BAB1_0476 promoter-luciferase transcriptional fusion grown to stationary phase in different media. Growth is also shown $(\boldsymbol{O})$.

proving that the $c f a$ deletion and the subsequent inability to synthesize CFA did not affect the ability to establish chronic infections in this model.

\section{Dysfunction of $B$. abortus cfa affects extracellular growth and survival}

The brucellae remain infectious for various periods of time on tissues from abortions, related tissues, fomites and soil, where they are exposed to dryness and subsequent osmotic stress and to the acidity that results from the activity of other microbiota. Thus, it is conceivable that, like in other bacteria, CFAs could contribute to persistence under these unfavourable extracellular conditions. To test this possibility, we first inoculated LB or LB- $340 \mathrm{mM}$ sucrose and measured growth. These experiments did not reveal significant differences between BAB-parental and BAB $\Delta c f a$ (data not shown). Then, we grew the bacteria to the stationary phase in LB and spotted aliquots of serial dilutions on LB agar with or without $340 \mathrm{mM}$ sucrose. We found that growth of $\mathrm{BAB} \Delta c f a$ but not of the revertant strain (generated after the excision of the mutator plasmid, and carrying the wild-type $c f a$ gene) was impaired on LB agar$340 \mathrm{mM}$ sucrose (Fig. 5a), and that the parental phenotype was partially restored by complementation of $\mathrm{BAB} \Delta c f a$ with plasmid pLPI-15 (not shown). We could not use a similar protocol to test growth on plates adjusted to an acidic $\mathrm{pH}$ because of the inability of BAB-parental and BAB $\Delta c f a$ to grow at $\mathrm{pH} 6$ or lower. Instead, we tested the survival after 48, 72, 96 and $120 \mathrm{~h}$ in Mcllvaine's buffer adjusted to $\mathrm{pH}$ 7,
6 or 5 by spotting aliquots of serial dilutions on standard TSA. We observed that BAB $\Delta c f a$ had a reduced ability to survive at $\mathrm{pH} 6$ and 5 and that the differences were most obvious at $120 \mathrm{~h}$ (Fig. 5b). This deficiency was partially repaired by complementation with plasmid pLPI-15 (not shown).

\section{DISCUSSION}

This research demonstrates that BAB1_0476 encodes the $B$. abortus CFA synthase that accounts for C19cyc (and $\mathrm{C} 17$ cyc) synthesis in this bacterium. Since C19cyc is also a major component of the lipids of all Brucella species analysed thus far (Coloe et al., 1984; De et al., 2008; Dees et al., 1981; Iriarte et al., 2004; Thiele et al., 1969; Thiele \& Schwinn, 1974; Vasiurenko et al., 1977) and all Brucella species carry an ORF identical or almost identical to BAB1_0476, it is likely that this conclusion also applies to those BAB1_0476 orthologues. Taking into account the presence of $c f a$ in all Brucella species, the large amounts of C19cyc produced, and the fact that synthesis of each C19cyc molecule requires one SAM and, therefore, three ATP (Cronan, 2002), it seems that C19cyc must confer some adaptive advantage to these pathogens.

As summarized in the Introduction, CFAs have been linked to an adaptation of the cell envelope to stressful environmental conditions, including those prevailing in the stationary phase, and indirect evidence suggests that at least low oxygen tension, nutrient limitation and acidic $\mathrm{pH}$ (a)

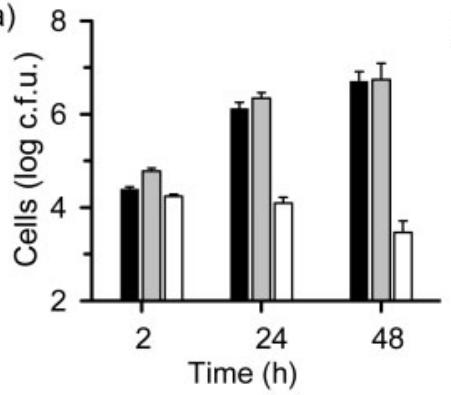

(b)

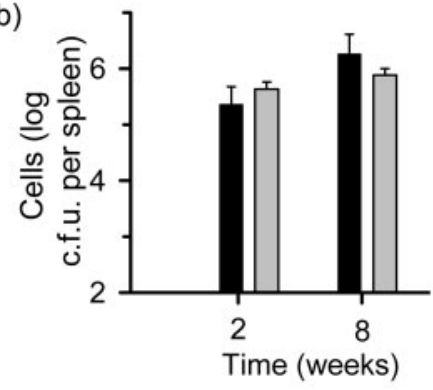

Fig. 4. The CFA deficiency does not alter the ability of $B$. abortus to multiply in RAW 264.7 macrophages (a) and in mice (b). Values are the mean \pm SEM of triplicate infections, and the results shown are representative of three independent experiments. Bars: black, BABparental; grey, BAB $\triangle c f a$; white, virB. 
(a)

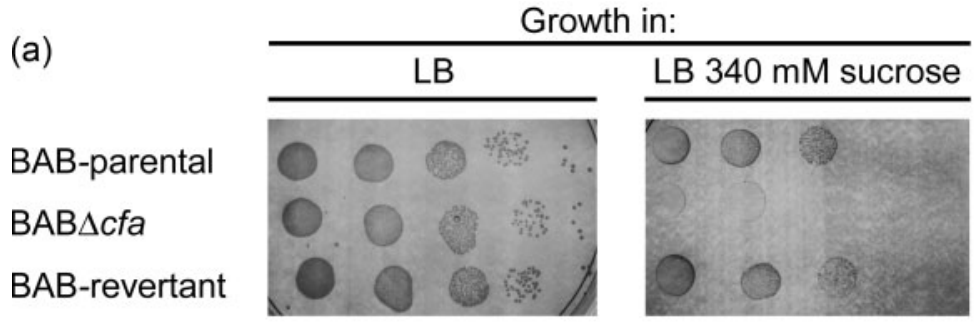

(b)

BAB-parental

$\mathrm{BAB} \Delta c f a$

BAB-revertant

Growth in standard TSB after $120 \mathrm{~h}$ of incubation in buffer at $\mathrm{pH}$ :

Fig. 5. B. abortus cfa is necessary for growth on hyperosmotic agar media (a) and for survival at acid pH (b). Serial dilutions of bacterial suspensions were made in saline and $10 \mu \mathrm{l}$ of each (from $10^{-4}$ to $10^{-8}$ ) was spotted on the indicated media and incubated at $37{ }^{\circ} \mathrm{C}$ for 7 days.

are encountered by brucellae in macrophages (JubierMaurin et al., 2004). On this basis, it has been hypothesized that Brucella cfa is a stationary phase gene and that it plays a role in intracellular life (Roop et al., 2003). Concerning the first hypothesis, our results show that expression of $B$. abortus $c f a$ occurs mostly during the exponential phase of growth. Although Brucella cfa is expressed during exponential phase and not during the stationary phase, this evidence cannot be interpreted to mean that the $\mathrm{C} 19 \mathrm{cyc}$ is synthesized only during exponential phase. In fact, C19cyc is a small proportion of the acyl chains of the free lipids present in the outer membrane blebs released by exponentially growing $B$. melitensis; $\mathrm{C} 18: 1$ is present in much larger amounts (Gamazo \& Moriyón, 1987). In E. coli, only a low proportion of unsaturated acyl chains are converted into CFAs during exponential growth (Grogan \& Cronan, 1997). In this bacterium, $c f a$ expression depends on RpoD ( $\sigma 70$; active throughout growth) and RpoS ( $\sigma 38)$, which regulates stationary phase genes (Wang \& Cronan, 1994). Moreover, there is a proteolytic degradation of CFA synthase at the beginning of stationary phase that makes its activity transitory (Chang et al., 2000). Thus, CFA accumulation in stationary phase cells is the outcome of the action of RpoS and lipid synthesis arrest (Wang \& Cronan, 1994). This indicates that CFA accumulation is a complex phenomenon compatible with $c f a$ expression in exponentially growing cells. With respect to the possibility that $\mathrm{C} 19 \mathrm{cyc}$ could be relevant in intracellular life, the ability of the $c f a$ mutant to multiply in murine macrophages and to establish chronic infections in mice seem to disprove this hypothesis, at least in these laboratory models. Indeed, disruption of this ORF completely abolished synthesis of cyclopropane fatty acids in vitro and, although the possibility that another CFA synthase could be activated in vivo cannot be completely excluded,
BAB1_0476 is the only ORF in the Brucella genomes that conserves both the SAM binding motif and all the residues necessary for CFA synthase activity. It is known that cyclopropane rings modulate innate immunity recognition of mycolic acids (Dao et al., 2008; Rao et al., 2005, 2006) and that $B$. abortus escapes early detection by innate immunity because of the reduced PAMP of envelope molecules with lipidic moieties (Barquero-Calvo et al., 2007). Although the presence of long acyl chains in these molecules is essential in PAMP reduction in Brucella (Lapaque et al., 2006; PalaciosChaves et al., 2011), our results suggest that C19cyc is not critical and that it can be substituted by $\mathrm{C} 18: 1$. This conclusion is consistent with the observation that $B$. abortus expresses $c f a$ at the same level in vitro as in baby hamster kidney cells (Viadas et al., 2010).

It has been postulated that the acidic $\mathrm{pH}$ of stomach represents a barrier when infection occurs orally in humans. If this is true, CFAs could play a role at this level. However, there is considerable evidence indicating that penetration of brucellae in humans (or ruminants) occurs through the oral mucosae rather than through the small or large intestine (for a discussion, see Gorvel et al., 2009). Like in B. abortus, mutation of the CFA synthases of the phylogenetic relative $S$. meliloti does not hamper intracellular life, and it has been suggested that CFAs could be useful during S. meliloti extracellular life (Saborido Basconcillo et al., 2009). A similar assumption can be made for $B$. abortus. Under natural conditions, these bacteria do not multiply significantly outside the hosts but can persist on tissues from abortions or related tissues, on materials contaminated with vaginal fluids, in milk and in dairy products. It has been known for a long time that, protected from direct sunlight and at temperatures below $55{ }^{\circ} \mathrm{C}$, Brucella is not easily destroyed: B. abortus can persist for 6-8 months on fetuses, 
for 3-4 months in faeces, for 1-3 months in soil, depending on the humidity, and for up to 4 days in milk at room temperature (Huddleson, 1943; Corbel, 2006). Indeed, the environmental conditions must be very different from those encountered in the endoplasmic reticulum-derived vacuolae where brucellae multiply normally. Undoubtedly, such extracellular conditions include acidity as a result of fermentations carried out by other micro-organisms, and the high tonicity associated with dryness of surfaces exposed to air. In this work, we have presented evidence showing that acidity and high osmolarity negatively affected the survival of a $B$. abortus cfa mutant. The fact that these observations were made on agar but could not be reproduced in broth indicates that additional factors must also be relevant. Whereas stirred broth provides homogeneous growth, micro-organisms are constrained to grow as colonies on structured products like agar media, many foodstuffs or contaminated surfaces. This results in a heterogeneous environment that causes an additional stress and slower growth rates, possibly because of the local depletion of oxygen and nutrients and accumulation of metabolites with altered pH gradients as a consequence (Theys et al., 2008). These conditions on structured media probably reflect more closely those on the materials that become contaminated when $B$. abortus is released in the environment. Thus, a reasonable hypothesis that is consistent with our in vitro results is that CFAs play a role in survival outside the hosts, thus favouring the possibility of transmission. This hypothesis could explain why an energetically costly function that does not play a role in intracellular life is conserved in this facultative intracellular parasite.

\section{ACKNOWLEDGEMENTS}

We thank Professor X. de Bolle (Unité de Recherche en Biologie Moleculaire, Facultes Universitaires Notre-Dame de la Paix, Belgique) for supervision of the construction of plasmid pSKoriTKmLuxAB. We also thank E. D. Babot and J. C. del Río for collaboration in GCMS analyses. Research at the laboratories of the authors is supported by the Ministerio de Ciencia y Tecnología of Spain (AGL2008-04514C03). Fellowship support for R. C.-Á., L. P.-C., Y.G.-R. and A.Z.-R from the Ministerio de Ciencia y Tecnología of Spain, Gobierno de Navarra and Friends of the University of Navarra is also acknowledged.

\section{REFERENCES}

Alton, G. G., Jones, L. M., Angus, R. D. \& Verger, J. M. (1988). Techniques for the Brucellosis Laboratory. Paris: INRA.

Arellano-Reynoso, B., Lapaque, N., Salcedo, S., Briones, G., Ciocchini, A. E., Ugalde, R., Moreno, E., Moriyón, I. \& Gorvel, J. P. (2005). Cyclic $\beta$-1,2-glucan is a Brucella virulence factor required for intracellular survival. Nat Immunol 6, 618-625.

Barkan, D., Rao, V., Sukenick, G. D. \& Glickman, M. S. (2010). Redundant function of $\mathrm{cmaA2}$ and mmaA2 in Mycobacterium tuberculosis cis cyclopropanation of oxygenated mycolates. J Bacteriol 192, 3661-3668.

Barquero-Calvo, E., Chaves-Olarte, E., Weiss, D. S., Guzmán-Verri, C., Chacón-Díaz, C., Rucavado, A., Moriyón, I. \& Moreno, E. (2007)
Brucella abortus uses a stealthy strategy to avoid activation of the innate immune system during the onset of infection. PLoS ONE 2, e631.

Bligh, E. G. \& Dyer, W. J. (1959). A rapid method of total lipid extraction and purification. Can J Biochem Physiol 37, 911-917.

Chang, Y. Y., Eichel, J. \& Cronan, J. E., Jr (2000). Metabolic instability of Escherichia coli cyclopropane fatty acid synthase is due to RpoHdependent proteolysis. J Bacteriol 182, 4288-4294.

Coloe, P. J., Sinclair, A. J., Slattery, J. F. \& Burke, D. (1984). Differentiation of Brucella ovis from Brucella abortus by gas-liquid chromatographic analysis of cellular fatty acids. J Clin Microbiol 19, 896-898.

Conde-Álvarez, R., Grilló, M. J., Salcedo, S. P., de Miguel, M. J., Fugier, E., Gorvel, J. P., Moriyón, I. \& Iriarte, M. (2006). Synthesis of phosphatidylcholine, a typical eukaryotic phospholipid, is necessary for full virulence of the intracellular bacterial parasite Brucella abortus. Cell Microbiol 8, 1322-1335.

Corbel, M. J. (2006). Brucellosis in Humans and Animals. Geneva: WHO Press.

Courtois, F., Guérard, C., Thomas, X. \& Ploux, O. (2004). Escherichia coli cyclopropane fatty acid synthase. Eur J Biochem 271, 4769-4778.

Cronan, J. E., Jr (2002). Phospholipid modifications in bacteria. Curr Opin Microbiol 5, 202-205.

Dao, D. N., Sweeney, K., Hsu, T., Gurcha, S. S., Nascimento, I. P., Roshevsky, D., Besra, G. S., Chan, J., Porcelli, S. A. \& Jacobs, W. R. (2008). Mycolic acid modification by the mmaA4 gene of $M$. tuberculosis modulates IL-12 production. PLoS Pathog 4, e1000081.

De, B. K., Stauffer, L., Koylass, M. S., Sharp, S. E., Gee, J. E., Helsel, L. O., Steigerwalt, A. G., Vega, R., Clark, T. A. \& other authors (2008). Novel Brucella strain (BO1) associated with a prosthetic breast implant infection. Clin Microbiol 46, 43-49.

Dees, S. B., Hollis, D. G., Weaver, R. E. \& Moss, C. W. (1981). Cellular fatty acids of Brucella canis and Brucella suis. J Clin Microbiol 14, 111112.

Dricot, A., Rual, J. F., Lamesch, P., Bertin, N., Dupuy, D., Hao, T., Lambert, C., Hallez, R., Delroisse, J. M. \& other authors (2004). Generation of the Brucella melitensis ORFeome version 1.1. Genome Res 14, 2201-2206.

Freer, E., Moreno, E., Moriyón, I., Pizarro-Cerdá, J., Weintraub, A. \& Gorvel, J. P. (1996). Brucella-Salmonella lipopolysaccharide chimeras are less permeable to hydrophobic probes and more sensitive to cationic peptides and EDTA than are their native Brucella sp. counterparts. J Bacteriol 178, 5867-5876.

Gamazo, C. \& Moriyón, I. (1987). Release of outer membrane fragments by exponentially growing Brucella melitensis cells. Infect Immun 55, 609-615.

Gerhardt, P. (1958). The nutrition of brucellae. Bacteriol Rev 22, 8198.

Glickman, M. S., Cox, J. S. \& Jacobs, W. R., Jr (2000). A novel mycolic acid cyclopropane synthetase is required for cording, persistence, and virulence of Mycobacterium tuberculosis. Mol Cell 5, 717-727.

Gómez-Miguel, M. J. \& Moriyón, I. (1986). Demonstration of a peptidoglycan-linked lipoprotein and characterization of its trypsin fragment in the outer membrane of Brucella spp. Infect Immun 53, 678-684.

Gorvel, J. P., Moreno, E. \& Moriyón, I. (2009). Is Brucella an enteric pathogen? Nat Rev Microbiol 7, 250, author reply 250.

Grilló, M. J., Manterola, L., de Miguel, M. J., Muñoz, P. M., Blasco, J. M., Moriyón, I. \& López-Goñi, I. (2006). Increases of efficacy as vaccine against Brucella abortus infection in mice by simultaneous inoculation with avirulent smooth $b v r S / b v r R$ and rough $w b k A$ mutants. Vaccine 24, 2910-2916. 
Grogan, D. W. \& Cronan, J. E., Jr (1997). Cyclopropane ring formation in membrane lipids of bacteria. Microbiol Mol Biol Rev 61, 429-441.

Hacker, S., Sohlenkamp, C., Aktas, M., Geiger, O. \& Narberhaus, F. (2008). Multiple phospholipid N-methyltransferases with distinct substrate specificities are encoded in Bradyrhizobium japonicum. J Bacteriol 190, 571-580.

Hallez, R., Letesson, J. J., Vandenhaute, J. \& De Bolle, X. (2007). Gateway-based destination vectors for functional analyses of bacterial ORFeomes: application to the Min system in Brucella abortus. Appl Environ Microbiol 73, 1375-1379.

Huddleson, I. F. (1943). Brucellosis in Men and Animals, Revised Edn. New York: The Commonwealth Fund.

Ingrosso, D., Fowler, A. V., Bleibaum, J. \& Clarke, S. (1989). Sequence of the D-aspartyl/L-isoaspartyl protein methyltransferase from human erythrocytes. Common sequence motifs for protein, DNA, RNA, and small molecule $S$-adenosylmethionine-dependent methyltransferases. J Biol Chem 264, 20131-20139.

Iñón de lannino, N., Briones, G. C., lannino, F. \& Ugalde, R. A. (2000). Osmotic regulation of cyclic 1,2- $\beta$-glucan synthesis. Microbiology 146, 1735-1742.

Iriarte, M., González, D., Delrue, R. M., Monreal, D., Conde-Álvarez, R., López-Goñi, I., Letesson, J. J. \& Moriyón, I. (2004). Brucella lipopolysacharide: structure, biosynthesis and genetics. In Brucella: Molecular and Cellular Biology, pp. 159-192. Edited by I. López-Goñi \& I. Moriyón. Wymondham, UK: Horizon Bioscience.

Jubier-Maurin, V., Loisel, S., Liautard, J. P. \& Köhler, S. (2004). The intramacrophagic environment of Brucella spp. and their replicative niche. In Brucella: Molecular and Cellular Biology, pp. 313-340. Edited by I. López-Goñi \& I. Moriyón. Wymondham, UK: Horizon Bioscience.

Kaneko, T., Nakamura, Y., Sato, S., Asamizu, E., Kato, T., Sasamoto, S., Watanabe, A., Idesawa, K., Ishikawa, A. \& other authors (2000). Complete genome structure of the nitrogen-fixing symbiotic bacterium Mesorhizobium loti. DNA Res 7, 331-338.

Lapaque, N., Takeuchi, O., Corrales, F., Akira, S., Moriyón, I., Howard, J. C. \& Gorvel, J. P. (2006). Differential inductions of TNF- $\alpha$ and IGTP, IIGP by structurally diverse classic and non-classic lipopolysaccharides. Cell Microbiol 8, 401-413.

Martínez de Tejada, G. \& Moriyón, I. (1993). The outer membranes of Brucella spp. are not barriers to hydrophobic permeants. J Bacteriol 175, 5273-5275.

Martínez de Tejada, G., Pizarro-Cerdá, J., Moreno, E. \& Moriyón, I. (1995). The outer membranes of Brucella spp. are resistant to bactericidal cationic peptides. Infect Immun 63, 3054-3061.

Moriyón, I. \& Berman, D. T. (1982). Effects of nonionic, ionic, and dipolar ionic detergents and EDTA on the Brucella cell envelope. J Bacteriol 152, 822-828.

Palacios-Chaves, L., Conde-Álvarez, R., Gil-Ramírez, Y., ZúñigaRipa, A., Barquero-Calvo, E., Chacón-Díaz, C., Chaves-Olarte, E., Arce-Gorvel, V., Gorvel, J. P. \& other authors (2011). Brucella abortus ornithine lipids are dispensable outer membrane components devoid of a marked pathogen-associated molecular pattern. PLOS ONE 6, e16030.

Rao, V., Fujiwara, N., Porcelli, S. A. \& Glickman, M. S. (2005). Mycobacterium tuberculosis controls host innate immune activation through cyclopropane modification of a glycolipid effector molecule. J Exp Med 201, 535-543.

Rao, V., Gao, F., Chen, B., Jacobs, W. R., Jr \& Glickman, M. S. (2006). Trans-cyclopropanation of mycolic acids on trehalose dimycolate suppresses Mycobacterium tuberculosis-induced inflammation and virulence. J Clin Invest 116, 1660-1667.

Roop, R. M., II, Gee, J. M., Robertson, G. T., Richardson, J. M., Ng, W. L. \& Winkler, M. E. (2003). Brucella stationary-phase gene expression and virulence. Annu Rev Microbiol 57, 57-76.

Roset, M. S., Ciocchini, A. E., Ugalde, R. A. \& Iñón de lannino, N. (2006). The Brucella abortus cyclic beta-1,2-glucan virulence factor with O-ester-linked succinyl residues. J Bacteriol 188, 5003-5013.

Saborido Basconcillo, L., Zaheer, R., Finan, T. M. \& McCarry, B. E. (2009). Cyclopropane fatty acyl synthase in Sinorhizobium meliloti. Microbiology 155, 373-385.

Scupham, A. J. \& Triplett, E. W. (1997). Isolation and characterization of the UDP-glucose $4^{\prime}$-epimerase-encoding gene, galE, from Brucella abortus 2308. Gene 202, 53-59.

Sieira, R., Comerci, D. J., Sánchez, D. O. \& Ugalde, R. A. (2000). A homologue of an operon required for DNA transfer in Agrobacterium is required in Brucella abortus for virulence and intracellular multiplication. J Bacteriol 182, 4849-4855.

Theys, T. E., Geeraerd, A. H., Verhulst, A., Poot, K., Van Bree, I., Devlieghere, F., Moldenaers, P., Wilson, D., Brocklehurst, T. \& Van Impe, J. F. (2008). Effect of $\mathrm{pH}$, water activity and gel microstructure, including oxygen profiles and rheological characterization, on the growth kinetics of Salmonella Typhimurium. Int J Food Microbiol 128, 67-77.

Thiele, O. W. \& Schwinn, G. (1973). The free lipids of Brucella melitensis and Bordetella pertussis. Eur J Biochem 34, 333-344.

Thiele, O. W. \& Schwinn, G. (1974). Bacterial ornithine lipids. Z Allg Mikrobiol 14, 435-443 (in German).

Thiele, O. W., Lacave, C. \& Asselineau, J. (1969). On the fatty acids of Brucella abortus and Brucella melitensis. Eur J Biochem 7, 393-396.

Vasiurenko, Z. P., Siniak, K. M., Korotich, A. S. \& Antonova, L. A. (1977). Fatty acid makeup of various Brucella species and its relationship to the culture medium. Zh Mikrobiol Epidemiol Immunobiol 8, 53-59 (in Russian).

Velasco, J., Bengoechea, J. A., Brandenburg, K., Lindner, B., Seydel, U., González, D., Zähringer, U., Moreno, E. \& Moriyón, I. (2000). Brucella abortus and its closest phylogenetic relative, Ochrobactrum spp., differ in outer membrane permeability and cationic peptide resistance. Infect Immun 68, 3210-3218.

Viadas, C., Rodriguez, M. C., Sangari, F. J., Gorvel, J. P., Garcia-Lobo, J. M. \& López-Goñi, I. (2010). Transcriptome analysis of the Brucella abortus BvrR/BvrS two-component regulatory system. PLoS ONE 5, e10216.

Wang, A. Y. \& Cronan, J. E., Jr (1994). The growth phase-dependent synthesis of cyclopropane fatty acids in Escherichia coli is the result of an $\operatorname{RpoS}(\mathrm{KatF})$-dependent promoter plus enzyme instability. Mol Microbiol 11, 1009-1017.

Edited by: D. L. Gally 